\title{
High lipid storage in vacoular forms of subtype 6 blastocystis sp. in ostrich
}

\author{
Hemalatha Chandrasekaran', Suresh Kumar Govind ${ }^{1 *}$, Chandrawathani Panchadcharam², Premaalatha Bathmanaban²,
} Kalyani Raman ${ }^{1}$ and Gaythri Thergarajan ${ }^{1}$

\begin{abstract}
Background: Blastocystis sp., a widely prevalent intestinal protozoan parasite is found in a wide range of animals, including humans. The possibility of zoonotic transmission to human from birds especially ostriches led us to investigate on the cross infectivity of Blastocystis sp. isolated from the ostrich feces as well as the phenotypic and subtype characteristics. There is a need to investigate this especially with the rising number of ostrich farms due to the growing global ostrich industry.

Findings: $100 \%$ of the ostriches were found to be positive for Blastocystis sp. using the in-vitro cultivation method. Transmission electron microscopy revealed high electron dense material in the central body of the vacoular forms. The membrane layer of the ostrich isolate was significantly $(p=0.003)$ thicker as compared to human isolate. Sudan staining revealed that this was lipid accumulation. We provide evidence for the first time, the existence of subtype 6 which has been previously reported only in pigs and cattle. Cysts, ranging from 3.0 to $7.0 \mu \mathrm{m}$ in diameter caused experimental infection in Sprague Dawley rats implicating that Blastocystis sp. isolated from ostriches exhibits low host specificity.
\end{abstract}

Conclusion: The study for the first time demonstrates that Blastocystis sp. subtype 6 do exist in ostriches and show high lipid storage in the vacuoles of the parasites. The study further provides evidence for potential zoonotic transmission in ostrich farms as Blastocystis subtype 6 can infect rats and the same subtype have been previously reported in humans.

Keywords: Blastocystis sp, Ultrastructure, Subtype, Host susceptibility

\section{Findings}

The increasing number of ostrich farms led us to investigate on the possibility of cross infectivity of Blastocystis sp. isolated from the ostrich feces as well elucidate phenotypic and subtype characteristics. 37 fresh fecal samples of ostriches (Struthio camelus) and fresh human fecal sample were collected from a local ostrich farm and from an asymptomatic individual infected with Blastocystis sp. respectively from one of the states in Malaysia and cultured using the in-vitro culture technique using Jones' medium and sequencedtagged site (STS) primer-polymerase chain reaction using ten sets of primers for subtype analysis. 100\% of the ostriches were found to be positive for Blastocystis sp.

\footnotetext{
* Correspondence: suresh@um.edu.my

${ }^{1}$ Department of Parasitology, Faculty of Medicine, University of Malaya, 50603 Kuala Lumpur, Malaysia

Full list of author information is available at the end of the article
}

using the in-vitro cultivation method. Transmission electron microscopy revealed high electron dense material in the central body of the vacoular forms. We provide evidence for the first time, the existence of subtype 6 in ostriches which showed high lipid storage. Cysts, ranging from 3.0 to $7.0 \mu \mathrm{m}$ in diameter caused experimental infection in Sprague Dawley rats implicating that Blastocystis sp. isolated from ostriches exhibits low host specificity. The study further provides evidence for potential zoonotic transmission in ostrich farms as Blastocystis subtype 6 can infect rats and the same subtype have been previously reported in humans.

\section{Background}

Blastocystis sp. is a widely prevalent intestinal protozoan parasite seen in a wide of a range of animals, including humans. Previous publications have shown Blastocystis 
sp. in non-human primates, birds, chickens, ducks, geese, ostriches, amphibians, reptiles, fish, arthropods and annelids [1-6].

There are of 17 distinct subtypes (ST1-ST17) seen in humans, non-human primates, other mammals and birds [7-9]. Although only some subtypes are described to be host specific especially subtype 6 which are commonly found in pigs and cattle, however most of the subtypes are shown to exhibit low host specificity [10] which may play a role in the cross-transmission between animal and human especially humans with histories of close association to pets or farm animals [11]. Transmission is through the fecal-oral route, though waterborne, food borne and sexual transmission have been reported [12-15].

Despite a few studies showing Blastocysis in ostriches [16-19], there has been none that has elucidated the ultrastructural details and subtype characterization. As ostrich farming industry is increasing worldwide including Malaysia due to its economic sale of its meat, feathers, oil and leather, it is vital to ascertain of the possible potential zoonotic transmission that can be transmitted to man. It is also possible that rats can acquire the infection by traveling to human dwelling. The study attempts to further elucidate information pertaining to ultrastructural, subtype and host susceptibility of Blastocystis sp. isolated from domestic ostriches.

\section{Materials and methods Ethical approval}

All animals used in this study were handled according to Institutional Animal Care and Use Committee (IACUC), University Malaya guidelines with the Reference. No: PAR/29/06/2012/LIL(R) and PAR/23/05/2013/HC(R). Human ethical approval for this study was obtained in accordance with University Malaya Medical Centre research policy with Reference. No: 926.7.

\section{Animals and management}

A local ostrich farm from one of the states in Malaysia was selected for the study. The farm practiced intensive type of management where the animals are confined in the same pen. Ostriches were kept in pairs or three's for breeding purpose in well-fenced housing pens. The animals were fed with quail layer mash, napier grass and given ad libitum water.

\section{Source of Blastocystis sp. isolates}

A total of thirthy-seven $(n=37)$ fresh fecal samples of ostriches (Struthio camelus) and fresh human fecal sample were collected from a local ostrich farm and from an asymptomatic individual infected with Blastocystis sp. respectively from one of the states in Malaysia. The samples were collected in stool collection container and were processed as soon after collection.

\section{Laboratory testing}

\section{In vitro cultivation of Blastocystis sp. isolates}

The parasites were isolated from the fecal samples of ostriches and human by in-vitro cultivation using Jones' medium supplemented with $10 \%$ heat-inactivated horse serum at $37^{\circ} \mathrm{C}$. Subsequently after isolation, the parasites were maintained in Jones' medium by consecutive sub-cultures every 3 to 4 days for at least one month prior to phenotypic, subtype and ultrastructural analysis $[6,20]$.

\section{Floatation method}

Approximately one gram of fresh feces, emulsified with saturated salt solution was then filtered through gauze into a centrifuge tube. Saturated salt solution was then later added up to the meniscus of test tube before lowering a coverslip onto the top of the tube. Coverslip was then lifted vertically up and placed onto a clean slide. Samples were observed under 10x objective lens of compound microscope for the presence of helminth ova, nematode eggs, coccidia oocysts and other parasites [21].

\section{Transmission electron microscopy}

Parasites isolated from ostriches and human were chosen for the ultrastructural studies. The contents of day 3 culture were washed three times using phosphate buffered saline (PBS) pH 7.4. The samples were centrifuged at 3000rpm for 5 minutes. The pelleted cells were re-suspended overnight in $2.5 \%$ glutaraldehyde in $0.1 \mathrm{M}$ sodium cacodylate buffer, $\mathrm{pH} 7.3$ at $4^{\circ} \mathrm{C}$, washed thoroughly with cacodylate buffer and post fixed for 30 min in $1 \%$ osmium tetroxide in cacodylate buffer. The fixed cells were dehydrated for 5 minutes in ascending series of ethanols $(30 \%, 50 \%, 70 \%, 80 \%, 90 \%$ and $100 \%)$ and embedded in epoxy resin. Semi-thin sections were stained with toluidine blue. Ultrathin sections were cut, contrasted with uranyl acetate and lead citrate and viewed using a transmission electron microscope (LEO Libra120) [22].

\section{Sudan Black B staining}

Parasites from day 3 culture of the ostrich and human isolates were smeared on a clean glass slide and immediately dried with a hair dryer at room temperature. Then the cells were fixed with $4 \%$ glutaraldehyde fixative solution in borate buffer, $\mathrm{pH} 7.6$ for 1 minute at $2-8^{\circ} \mathrm{C}$ with gentle agitation followed by thorough rinsing in deionized water. The cells were then stained with Sudan Black B reagent by immersing slides for 5 minutes with intermittent agitation. The cells were then 
Table 1 List of sequenced-tagged site (STS) primers

\begin{tabular}{|c|c|c|c|c|}
\hline Subtype & STS primer & Product size (bp) & Sequence of forward (F) and reverse (R) primer $\left(5^{\prime}-3^{\prime}\right)$ & Genebank accession no. \\
\hline \multirow[t]{2}{*}{1} & \multirow[t]{2}{*}{ SB82 } & \multirow[t]{2}{*}{462} & F-TCTTGCTTCATCGGAGTC & \multirow[t]{2}{*}{ AF166085 } \\
\hline & & & R-CCTTCTCGCAGTTCTTTATC & \\
\hline \multirow[t]{2}{*}{1} & \multirow[t]{2}{*}{ SB83 } & \multirow[t]{2}{*}{351} & F-GAAGGACTCTCTGACGATGA & \multirow[t]{2}{*}{ AF166086 } \\
\hline & & & R-GTCCAAATGAAAGGCAGC & \\
\hline \multirow[t]{2}{*}{2} & \multirow[t]{2}{*}{ SB155 } & \multirow[t]{2}{*}{650} & F-ATCAGCCTACAATCTCCTC & \multirow[t]{2}{*}{ AF166087 } \\
\hline & & & R-ATCGCCACTTCTCCAAT & \\
\hline \multirow[t]{2}{*}{3} & \multirow[t]{2}{*}{ SB227 } & \multirow[t]{2}{*}{526} & F-TAGGATTTGGTGTTTGGAGA & \multirow[t]{2}{*}{ AF166088 } \\
\hline & & & R-TTAGAAGTGAAGGAGATGGAAG & \\
\hline \multirow[t]{2}{*}{3} & \multirow[t]{2}{*}{ SB228 } & \multirow[t]{2}{*}{473} & F-GACTCCAGAAACTCGCAGAC & \multirow[t]{2}{*}{ AF166089 } \\
\hline & & & R-TCTTGTTTCCCCAGTTATCC & \\
\hline \multirow[t]{2}{*}{3} & \multirow[t]{2}{*}{ SB229 } & \multirow[t]{2}{*}{631} & F-CACTGTGTCGTCATTGTTTTG & \multirow[t]{2}{*}{ AF166090 } \\
\hline & & & R-AGGGCTGCATAATAGAGTGG & \\
\hline \multirow[t]{2}{*}{4} & \multirow[t]{2}{*}{ SB332 } & \multirow[t]{2}{*}{338} & F-GCATCCAGACTACTATCAACATT & \multirow[t]{2}{*}{ AF166091 } \\
\hline & & & R-CCATITTCAGACAACCACTTA & \\
\hline \multirow[t]{2}{*}{5} & \multirow[t]{2}{*}{ SB340 } & \multirow[t]{2}{*}{704} & F-TGTTCTTGTGTCTTCTCAGCTC & \multirow[t]{2}{*}{ AY048752 } \\
\hline & & & R-TTCTITCACACTCCCGTCAT & \\
\hline \multirow[t]{2}{*}{6} & \multirow[t]{2}{*}{ SB336 } & \multirow[t]{2}{*}{317} & F-GTGGGTAGAGGAAGGAAAACA & \multirow[t]{2}{*}{ AY048751 } \\
\hline & & & R-GAACAAGTCGATGAAGTGAGAT & \\
\hline$\overline{7}$ & SB337 & 487 & F-GTCTTTCCCTGTCTATTCTGCA & AY048750 \\
\hline & & & R-AATTCGGTCTGCTTCTTCTG & \\
\hline
\end{tabular}

rinsed 3 to 5 times in $70 \%$ ethanol followed by a thorough rinsing in distilled water. After rinsing, the cells were counterstained in haematoxylin solution for 5 minutes followed by thorough rinsing in tap water. Slides were then examined under a conventional Olympus microscope equipped with an immersion oil objective lens (100x) for the presence of black droplets in the central vacuole indicating positive reactions. The samples were stained using a commercial solution, Sudan Black B Staining System (Sigma Aldrich, Germany) according to the recommendations of the manufacturer.

\section{Molecular detection}

\section{Genomic DNA preparation}

DNA was extracted from the culture sample of all 37 ostrich isolates and a human isolate using QIAamp DNA Stool Mini Kit (QIAGEN, Hilden, Germany) according to the manufacturer's protocol [4].

\section{Subtyping of Blastocystis sp. isolates}

All 37 parasite isolates were subjected to sequencedtagged site (STS) primer-polymerase chain reaction using the following ten sets of primers [4] (Table 1). Two to five $\mu$ l of DNA preparations were used to amplify the genomic sequences in a $20 \mu \mathrm{l}$ reaction containing $0.5 \mathrm{mM}$ of the dNTPs, $0.5 \mathrm{mM}$ of each primer, $1 \times$ PCR buffer (75 mM Tris-HCL, pH 8.8, $20 \mathrm{mM}\left(\mathrm{NH}_{4}\right)_{2} \mathrm{SO}_{4}$ and $0.01 \%$ Tween 20), $2.5 \mathrm{mMm} \mathrm{MgCl}_{2}$ and $1 \mathrm{U}$ Taq
DNA Polymerase (recombinant) (FERMENTAS, USA). PCR conditions consisted of 1 cycle of initial denaturing at $94^{\circ} \mathrm{C}$ for 3 minutes, followed by 30 cycles including denaturing at $94^{\circ} \mathrm{C}$ for $30 \mathrm{~s}$, annealing at $57^{\circ} \mathrm{C}$ for $30 \mathrm{~s}$ and extending at $72^{\circ} \mathrm{C}$ for 1 minute, and an additional cycle with a $10 \mathrm{~min}$ chain elongation at $72^{\circ} \mathrm{C}$ (thermocycler Eppendorf, Germany). The amplification products were electrophoresed in 1.5\% agarose gels (PROMEGA USA) and Tris-Borate-EDTA buffer. Gels were stained with ethadium bromide and photographed using ultra-violet gel documentation system (Uvitec, United Kingdom). The PCR amplication for each primer pair was repeated thrice for each isolate [4]. The classification of the subtypes for each Blastocystis sp. isolate was based on the standard terminology [8].

\section{In vivo study}

The cysts, isolated from fresh fecal material of two respective ostriches, collected in separate tubes by using Ficoll Paque method were made to a concentration of $10^{5}$ of cysts $/ \mathrm{ml}$. This was then inoculated orally, using

Table 2 Prevalence of Blastocystis sp. in ostrich isolates

\begin{tabular}{llc}
\hline Sex of host & $\begin{array}{l}\text { Number of } \\
\text { animals }\end{array}$ & $\begin{array}{l}\text { Number of animals } \\
\text { detected positive }\end{array}$ \\
\hline Male & 20 & 20 \\
\hline Female & 17 & 17 \\
\hline
\end{tabular}




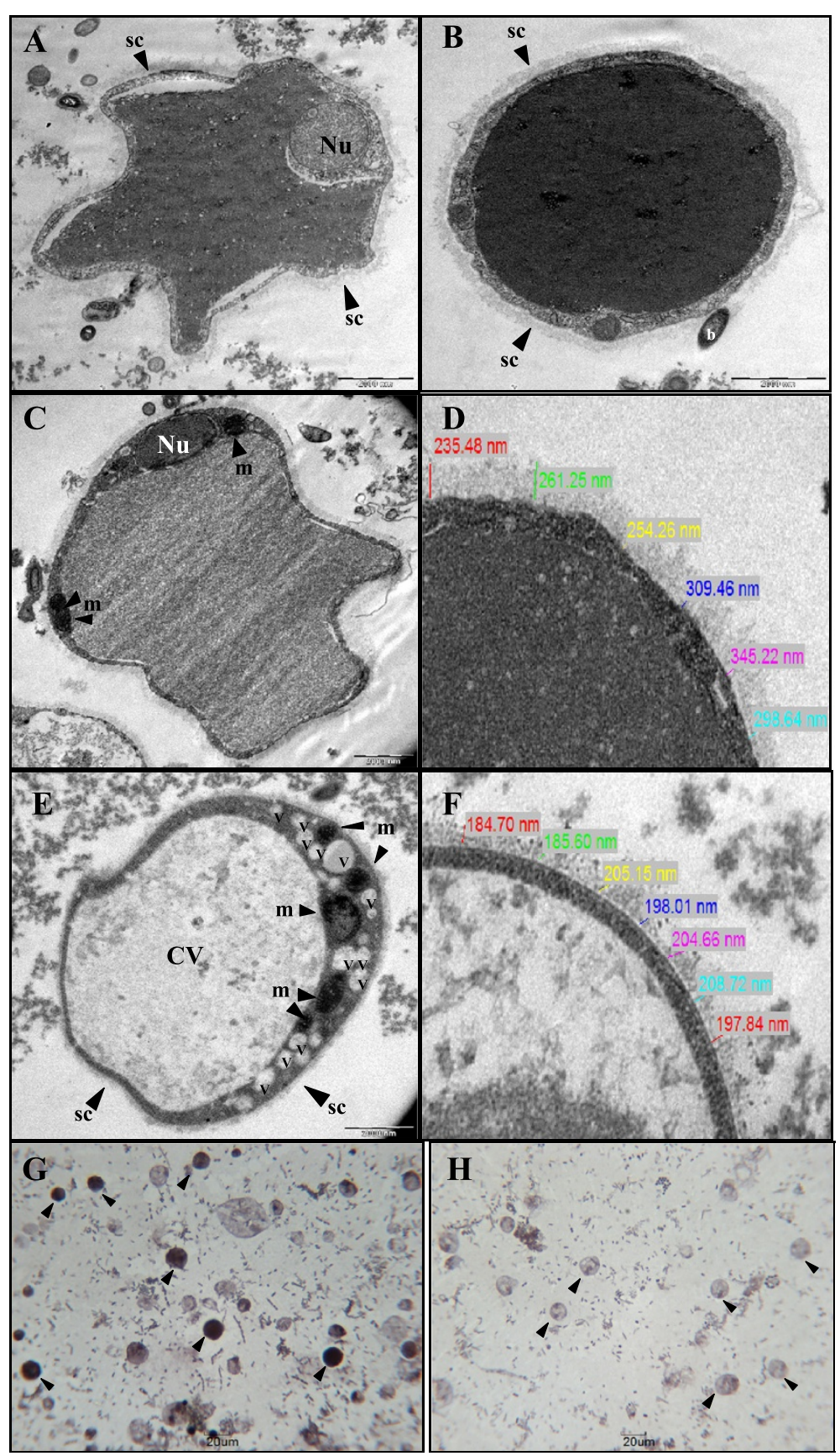

Figure 1 Comparison of Transmission electron microscopy and light microscopy of Blastocystis sp. for ostrich isolates and human isolates. A: Transmission electron micrograph showing an irregular shape Blastocystis sp. with a prominent nucleus (Nu) B: A thick, compact surface coat $(s c)$ is seen to surround the cell when examined by transmission electron microscopy. A high electron dense area was observed in the central vacuole $(C V$. C: Numerous mitochondria $(m)$ were seen in the Blastocystis sp. cells of the ostrich isolates. D: Higher magnification of Blastocystis sp. membrane in the ostrich faecal culture. E: A multi-vacoulated form (v) of Blastocystis sp. in human faecal culture with multiple mitochondria present in the cytoplasm. F: Higher magnification of Blastocystis sp. membrane in the human faecal culture. Note: the cell membrane of Blastocystis sp. in ostrich and human isolates were 235.48 to $345.22 \mathrm{~nm}$ and 184.70 to $208.72 \mathrm{~nm}$, respectively. G: Light microscopic images of Blastocystis sp. isolated on day 3 of ostrich faecal culture stained with Sudan Black B. Positive reactions are seen as dark droplets in the central vacuole. Note: dark droplets (arrows). $\mathbf{H}$ : Light microscopic images of Blastocystis sp. isolated on day 3 of human faecal culture stained with Sudan Black B. No reactions were observed in the central vacuole. 
Table 3 Statistical comparison of membrane thickness of Blastocystis sp. isolated from ostrich and human (p< 0.05 )

\begin{tabular}{lllcc}
\hline & Organism & Range & Means \pm S.D & p-value \\
\hline Thickness of membrane $(\mathbf{n m})$ & Ostrich & $235.48-345.22$ & $284.05 \pm 40.91$ \\
\cline { 2 - 4 } & Human & $184.70-208.72$ & $197.81 \pm 9.49$ \\
\hline
\end{tabular}

20G feeding needle of 1.5 inch length into nine Sprague Dawley rats. All the 9 rats were divided into two respective groups, Group A of 5 rats and Group B of 4 rats and were anesthetized before inoculation of respective strains. Stools were collected from the rats to examine for the presence of Blastocystis sp., two days of post-inoculation [23]. Samples found positive for Blastocystis sp. were then subjected to subtyping.

\section{Statistical analysis}

Statistical analysis was carried out using IBM $^{\odot}$ SPSS $^{\odot}$ Statistics Version 21. Independent Students t-test was used to assess the differences in the membrane thickness of Blastocystis sp. isolated from ostrich and human. A value of $\mathrm{p}<0.05$ is considered statistically significant.

\section{Results}

Prevalence

$100 \%$ of 37 ostriches were found to be positive for Blastocystis sp. No other parasites were seen. However all ostriches appeared healthy without any symptoms. Direct microscopy of fecal smears revealed 8-12 Blastocystis sp. under 40x magnification (Table 2).

\section{Ultrastructural studies of Blastocystis sp.}

Transmission electron micrographs of Blastocystis sp. showed slight irregular in shape with a thick and compact surface coat seen to be surrounding the cell (Figure 1A). Bacteria were occasionally seen to be adhering to the surface coat (Figure 1B). High electron dense material was observed in the central vacuole with the presence of two prominent nuclei in most of the parasites (Figure 1B). Numerous mitochondria were seen in the Blastocystis sp. cells of the ostrich isolates (Figure 1C). Meanwhile, Blastocystis sp. in human fecal culture (Figure 1E) illustrates a multi-vacoulated form of this organism with multiple mitochondria present in the cytoplasm and a clear, large central vacuole (CV). The membrane layer of the ostrich isolate (Figure 1D) was significantly $(\mathrm{p}=0.003)$ thicker, $284.05 \pm 40.91 \mathrm{~nm}$ (range, 235.48 to $345.22 \mathrm{~nm}$ ) as compared to human isolate (Figure 1F), $197.81 \pm 9.49 \mathrm{~nm}$ (range, 184.70 to $208.72 \mathrm{~nm}$ ) (Table 3). Parasites from day 3 cultures showed ostrich isolate stained with Sudan Black B, positive reactions were observed in the central vacuole of Blastocystis sp. (Figure 1G) meanwhile no reactions were observed in the central vacuole of the Blastocystis sp. from the day 3 human isolate (Figure $1 \mathrm{H}$ ). Positive reactions are seen as dark droplets in the central vacoule (CV).

\section{Subtyping of Blastocystis sp.}

Using sequenced-tagged site primer-PCR, 14 of the ostrich isolates were confirmed to be subtype 6 (Figure 2). Meanwhile, the subtype of remaining 23 isolates when amplified with PCR using sequence-tagged site (STS) revealed no bands when assessed using primers for subtypes 1 to 7 .

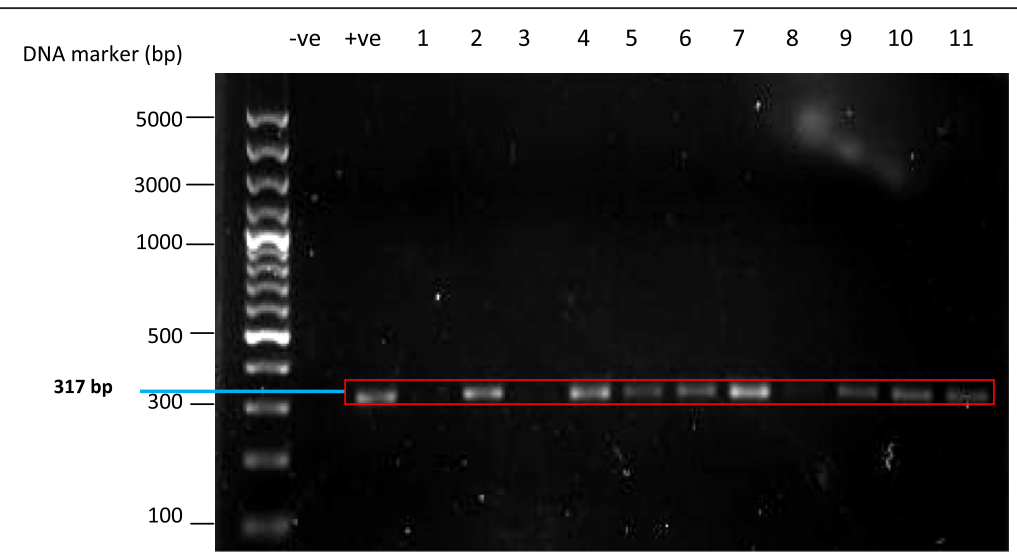

Figure 2 Examples of polymerase chain reaction (PCR) products from isolates of Blastocystis sp. amplified by sequenced-tagged site (STS) primers. Lane 1, DNA size markers of a 100-bp DNA ladder plus; Lane 2, negative control; Lane 3, positive control; Lane 5, Lanes 7-10, Lanes 12-14; subtype 6 (317 bp). 


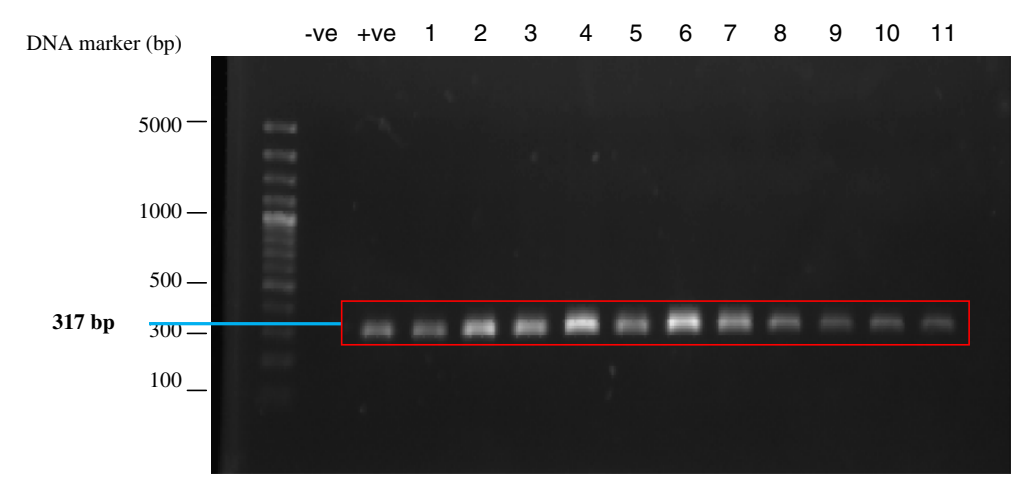

Figure 3 Examples of polymerase chain reaction (PCR) products from isolates of Blastocystis sp. from the cross-infection in vivo study amplified by sequenced-tagged site (STS) primers. Lane 1, DNA size markers of a 100-bp DNA ladder plus; Lane 2, negative control; Lane 3, positive control; Lanes 4-5, Blastocystis sp. cyst of ostrich isolate (inoculum); Lane 6-14, Blastocystis sp. isolates of infected rats; subtype 6 (317 bp).

\section{In vivo study}

The Blastocystis cysts isolated from fresh fecal samples of ostriches ranged from 3.0 to $7.0 \mu \mathrm{m}$ in diameter. These cysts caused experimental infection in Sprague Dawley rats. Blastocystis sp. was detected in feces of experimentally infected Sprague Dawley rats, two days of postinoculation. The rats infected with ostrich isolate were identified to be subtype 6 which was similar to the strain of the inoculum when amplified with PCR using sequencetagged site (STS) primers (Figure 3).

\section{Discussion}

A high prevalence of Blastocystis infection with 100\% positive in the ostrich population indicates that this organism is commonly found in this avian. Ultrastructural studies concurred with the previous studies [24,25]. Most of the cells appeared to be rounded or slightly irregular in shape with a thick and compact surface coat seen to be surrounding the cell. One or more nuclei with numerous mitochondria were commonly observed in the cytoplasm of the organism [5]. The outer membrane of ostrich isolate was observed to be significantly thicker when compared to human isolate possibly conferring greater resistance in non-conducive environments. The most distinguishing characteristic was the presence of high electron dense material within the vacuolar forms of parasites on day 3 cultures. Sudan Black B revealed in more than $50 \%$ of the parasites dark stains in portions of the central vacuole indicating the presence of neutral lipid [26]. The study confirms that Blastocystis sp. from ostrich isolates uses the vacuolar forms to store lipids.

Although subtype 6 was seen in livestock animals [9] especially in pigs and cattle [27] this is the first study to demonstrate subtype 6 seen in ostrich isolates when amplified with polymerase chain reaction using sequence-tagged site (STS) primers (Figure 2). Roberts et al. [19] showed that 6 out of 10 ostriches examined for Blastocystis sp. were subtype 7 . In the present study, the Blastocystis cysts of ostrich isolate were able to cause experimental infection in Sprague Dawley rats (Figure 3) as evidenced by the subtype 6 seen in the stools of infected rats which were prior negative for Blastocystis sp. Subtype 6 have been shown to be seen in humans [28] and therefore can be postulated that the ostrich farms with wild rats can be a reservoir for human infections since Blastocystis sp. exhibits low host specificity.

\section{Conclusion}

The present study is the first to elucidate the ultrastructural, subtype, and host susceptibility of Blastocystis $\mathrm{sp}$. isolated from ostriches in order to determine the true pathogenicity of this zoonotic parasite which is known to be a potential source for cross-transmission between animal and humans especially when in close association.

\section{Competing interests}

The authors declare that they have no competing interests.

\section{Authors' contributions}

$H C, S K G, C P, P B, K R$, and GT was involved in all phases of the study, including the conceptual framework and the design of the experiment, data collection and analysis, interpretation of results. HC wrote up the manuscript and all authors read and approved the finalized manuscript.

\section{Acknowledgements}

The authors would like to thank our lab colleagues and all the staffs at the Department of Parasitology, University of Malaya and Parasitology section, Veterinary Research Institute Ipoh and all the farmers involved in this study for their continued support and cooperation. This study was entirely supported by High Impact Research Grant (UM.C/HIR/MOHE/MED/44) and Postgraduate Research Grant (PV133-2012A) from University of Malaya.

\section{Author details}

'Department of Parasitology, Faculty of Medicine, University of Malaya, 50603 Kuala Lumpur, Malaysia. ${ }^{2}$ Veterinary Research Institute Ipoh, 59 Jalan Sultan Azlan Shah, 31400 Ipoh, Perak, Malaysia. 
Received: 21 May 2014 Accepted: 30 September 2014

Published online: 30 October 2014

\section{References}

1. Stenzel DJ, Boreham PF: Blastocystis hominis revisited. Clin Microbiol Rev 1996, 9:563-584

2. Konig G, Muller HE: Blastocystis hominis in animals: incidence of four serogroups. Zentralbl Bakteriol 1997, 286:435-440.

3. Belova LM, Krylov MV: The distribution of Blastocystis according to different systematic groups of hosts. Parazitologiia 1998, 32:268-276.

4. Yoshikawa H, Wu Z, Kimata I, Iseki M, Ali IK, Hossain MB, Zaman V, Haque R, Takahashi Y: Polymerase chain reaction-based genotype classification among human Blastocystis hominis populations isolated from different countries. Parasitol Res 2004, 92:22-29.

5. Stenzel DJ, Cassidy MF, Boreham PFL: Morphology of Blastocystis sp. from domestic birds. Parasitol Res 1994, 80:131-137.

6. Farah HMT, Chandrawathani P, Mohd ZSN, Suresh KG, Hemalatha C, Premaalatha B: A preliminary study of Blastocystis sp. isolated from chicken in Perak and Selangor, Malaysia. MJVR 2014, 5:21-25.

7. Noel C, Dufernez F, Gerbod D, Edgcomb VP, Delgado VP, Ho LC, Viscogliosi E: Molecular phylogenies of Blastocystis isolates from different hosts-implications for genetic diversity, identification of species, and zoonosis. J Clin Microbiol 2005, 43:348-355.

8. Stensvold CR, Suresh KG, Tan KS, Thompson RC, Traub RJ, Viscogliosi E, Clark CG: Terminology for Blastocystis subtypes-a consensus. Trends Parasitol 2007, 23:93-96.

9. Alfellani MA, Taner MD, Jacob AS, Imeede CA, Yoshikawa H, Stensvold CR, Clark CG: Genetic diversity of Blastocystis in livestock and zoo animals. Protist 2013, 164:497-509.

10. Yoshikawa H, Abe N, Iwasawa M, Kitano S, Nagano I, Wu Z, Takahashi Y: Genomic analysis of Blastocystis hominis strains isolated from two long-term health care facilities. J Clin Microbiol 2000, 38:1324-1330.

11. Doyle PW, Helgason MM, Mathias RG, Proctor EM: Epidemiology and pathogenicity of Blastocystis hominis. J Clin Microbiol 1990, 28:116-121.

12. Nimri L, Raymound B: Intestinal colonization of symptomatic and asymptomatic schoolchildren with Blastocystis hominis. J Clin Microbiol 1994, 32:2865-2866.

13. Casemore DP: Foodborne protozoal infection. Lancet 1990, 336:1427-1432.

14. Garavelli P, Orsi P, Scaglione L: Blastocystis hominis infection during AIDS. Lancet 1988, 332:1364

15. Llibre JM, Tor J, Manterola JM, Carbonell C, Foz M: Blastocystis hominis chronic diarrhoea in AIDS patients. Lancet 1989, 333:221.

16. Yamada M, Yoshikawa H, Tegoshi T, Matsumoto Y, Yoshikawa T, Shiota T, Yoshida Y: Light microscopical study of Blastocystis spp. in monkeys and fowls. Parasitol Res 1987, 73:527-531.

17. Pakandl M: Blastocystis sp. from pigs-ultrastructural changes occurring during polyxenic cultivation in Iscove's modified Dulbecco's medium. Parasitol Res 1999, 85:743-748.

18. Ponce GF, Herrera S, Castro AT, Garcia DB, Martinez DRA: Parasites from farmed ostriches (Struthio camelus) and rheas (Rhea Americana) in Europe. Vet Parasitol 2002, 107:137-160.

19. Roberts T, Stark D, Harkness J, Ellis J: Subtype distribution of Blastocystis isolates from a variety of animals from New South Wales, Australia. Vet Parasitol 2013, 196:85-89.

20. Suresh $\mathrm{K}, \mathrm{Ng} \mathrm{GC}, \mathrm{Ho} \mathrm{LC}$, Yap EH, Singh M: Differentiation of the various stages of Blastocystis hominis by acridine orange staining. Int J Parasitol 1994, 24:605-606.

21. Norakmar I, Chandrawathani P, Nurulaini R, Zawida Z, Premaalatha B, Imelda Lynn V, Adnan M, Jamnah O, Zaini CM: Prevalence of helminthiasis in relation to climate in small ruminants in Perak in 1998 and 2008. MJVR 2010, 1:37-44.

22. Tan TC: Predominance of amoeboid forms of Blastocystis hominis in isolates from symptomatic patients. Parasitol Res 2006, 98:189-193.

23. Suresh K, Ng GC, Ramachandran NP, Ho LC, Yap EH, Singh M: In vitro encystment and experimental infections of Blastocystis hominis. Parasitol Res 1993, 79:456-460.

24. Tan KS: Blastocystis in humans and animals: new insights using modern methodologies. Vet Parasitol 2004, 126:121-144.

25. Yoshikawa H, Wu Z, Howe J, Hashimoto T, Geok-Choo NG, Tan KS: Ultrastructural and phylogenetic studies on Blastocystis isolates from cockroaches. J Eukaryot Microbiol 2007, 54:33-37.
26. Yoshikawa H, Satoh J, Enose Y: Light and electron microscopic localization of lipids in Blastocystis hominis. J Electron Microsc 1995, 44:100-103.

27. Stensvold CR, Alfellani MA, Norskov LS, Prip K, Victory EL, Maddox C, Clark CG: Subtype distribution of Blastocystis isolates from synanthropic and zoo animals and identification of a new subtype. Int J Parasitol 2009, 39:473-479.

28. Stensvold CR, Lewis HC, Hammerum AM, Porsbo LJ, Nielsen SS, Olsen KEP, Molbak K: Blastocystis: unravelling potential risk factors and clinical significance of a common but neglected parasite. Epidemiol Infect 2009, 137:1655-1663.

doi:10.1186/s13071-014-0469-7

Cite this article as: Chandrasekaran et al:: High lipid storage in vacoular forms of subtype 6 blastocystis sp. in ostrich. Parasites \& Vectors 2014 7:469.

\section{Submit your next manuscript to BioMed Central and take full advantage of:}

- Convenient online submission

- Thorough peer review

- No space constraints or color figure charges

- Immediate publication on acceptance

- Inclusion in PubMed, CAS, Scopus and Google Scholar

- Research which is freely available for redistribution 